CWRU-P14-1995

\title{
Perturbed Electroweak Strings and Fermion Zero Modes
}

\author{
Hong Liu and Tanmay Vachaspati \\ Physics Department \\ Case Western Reserve University \\ Cleveland $\mathrm{OH}$ 44106-7079.
}

(July 18, 2018)

\begin{abstract}
The effect of perturbations of electroweak strings on quark zero modes is studied in $2+1$ and $3+1$ dimensions. As first discovered by Naculich, it is found that the bosonic perturbations that destabilize the string give a mass to the zero modes and also lift their degeneracy. The effect of the zero modes on the stability of the string is discussed qualitatively and we argue that the fermionic vacuum instability found by Naculich should lead to a distortion of the bosonic string but not be responsible for decay.
\end{abstract}




\section{INTRODUCTION}

Over the last few years there has been considerable interest in electroweak $Z$-strings [1:2]. These are flux tube solutions to the classical equations of motion of the bosonic sector of the standard electroweak model. Recently, interest has turned to the effect of fermions on the string.

The first issue in including fermions is the effect of the string on the fermion spectrum. While no one has found the full spectrum, it is well known that the mass spectrum contains zero energy solutions (zero modes) for all massive fermions in the standard model [3]. The zero modes lead to fermionic superconductivity of the $Z$-string.

The second issue in this research program and also one that we address in this paper, is to study the backreaction of fermions on the $Z$-string. Our analysis follows the recent work of Naculich [4] who has discussed the effects of fermions on the stability of an infinite $Z$-string. The strategy that is adopted is to perturb the string background and study the effect that this has on the fermion spectrum and on the fermion ground state energy. This energy contribution is then included in the total energy and tells us if the fermionic vacuum contributions to the energy are (i) first or second order in the perturbations, and, (ii) if they help to stabilize (destabilize) the string.

In this paper, we consider the back reaction of lepton and quark zero modes on $Z$-strings and recover some of Naculich's results. Our techniques and those used in Ref. [4] differ in two respects. The first difference is in our computation of the shift in the fermion energy levels due to perturbations in the bosonic fields. Naculich employs the standard result of second order perturbation theory in this computation in which a sum over a complete set of states needs to be done. The use of the standard result does not seem appropriate because the complete spectrum of the fermion modes in the string background is not known - only

the zero modes are known. So we do not use the standard result and solve the perturbed equations of motion directly. A second (minor) difference in our techniques is that we have included all possible perturbations and not just the perturbation in the Higgs field.

The result that the fermionic vacuum destabilizes the $Z$-string in [4] is due to an infrared logarithmic divergence arising from low momentum modes propagating along the string. In the $2+1$ dimensional case, such modes are absent and it is of some interest to consider the effect of the fermionic vacuum (and positive energy fermions) on the string. With this in mind, we first consider fermions on perturbed strings in $2+1$ dimensions and subsequently consider the $3+1$ case. But we find that it is not possible to evaluate the backreaction of fermions on the string because the full spectrum of fermion modes is not known. Only the zero mode is known, and, in $2+1$ dimensions, this is only one mode from an infinity of modes.

While we are not able to evaluate the backreaction quantitatively, we give an argument why the backreaction of fermions should lead to a distorted string solution but not be responsible for string decay. This follows once we realize that the calculations that have been done for the electroweak string can also be carried out for low energy topological strings that can terminate in superheavy magnetic monopoles. Once again, the fermionic vacuum will destabilize the topological string. But, in this case, the topology in the model assures us that the fermionic vacuum will not cause the string to decay. Hence, a distorted string solution must exist. The observation in [5] that the electroweak string can arise for 
topological reasons if the electroweak symmetry breaking occurred in stages suggests that the distorted string solution will also exist in the electroweak model.

We can repeat Naculich's arguments and consider the effect of fermions on perturbed topological strings. This, once again, for very general reasons, leads to an "instability". But now the topology indicates that the fermions will only distort the string and not be responsible for string decay.

While the present analysis deals with the $Z$-string, we would like to mention that fermionic effects on analogous vortices present in ${ }^{3} \mathrm{He}$ [6 have also been studied. Makhlin and Volovik [7] find that fermions distort the structure of the vortices. In future, we can expect that this distortion might even be observed in the laboratory [8].

In Section II, we review past results and set up our notation. In Section III, we outline the calculation that ideally needs to be carried out to determine the back-reaction of fermions on strings. In Section IV we find the effects of the perturbations on strings on the fermion zero modes in $2+1$ dimensions. Section $\mathrm{V}$ extends this calculation to $3+1$ dimensions and we discuss the fermion backreaction in Section VI. The Appendix contains a simple explicit proof that fermion zero modes exist on perturbed Nielsen-Olesen strings in the AbelianHiggs model. Though this follows from the application of an index theorem [9], our proof is an alternate way of seeing that the zero modes exist for topological rather than dynamical reasons.

\section{REVIEW OF $Z$-STRING AND FERMION ZERO MODES}

We consider the standard electroweak model,

$$
\begin{gathered}
\mathcal{L}=\mathcal{L}_{\text {Boson }}+\mathcal{L}_{\text {Fermion }} \\
\mathcal{L}_{\text {Boson }}=-\frac{1}{4} W_{\mu \nu}^{a} W^{a \mu \nu}-\frac{1}{4} F_{\mu \nu} F^{\mu \nu}+\left|D_{\mu} \Phi\right|^{2}-\lambda\left(\Phi^{\dagger} \Phi-\frac{\eta^{2}}{2}\right)^{2}
\end{gathered}
$$

where

$$
\Phi=\left(\begin{array}{c}
\phi_{1} \\
\phi_{0}
\end{array}\right), \quad D_{\mu} \Phi=\left(\partial_{\mu}-\frac{i}{2} g \tau^{a} W_{\mu}^{a}-\frac{i}{2} g^{\prime} B_{\mu}\right) \Phi
$$

and $W_{\mu \nu}^{a}$ and $F_{\mu \nu}$ are the corresponding field strength tensors for $W_{\mu}^{a}$ and $B_{\mu}$. Also define,

$$
\begin{gathered}
\alpha \equiv \sqrt{g^{2}+g^{\prime 2}}, \quad \tan \theta_{w} \equiv \frac{g^{\prime}}{g}, \quad e \equiv g \sin \theta_{w} \\
Z_{\mu} \equiv \cos \theta_{w} W_{\mu}^{3}-\sin \theta_{w} B_{\mu}, \quad A_{\mu} \equiv \sin \theta_{w} W_{\mu}^{3}+\cos \theta_{w} B_{\mu}
\end{gathered}
$$

where, as is conventional, the vacuum is chosen to be $\Phi_{v a c}^{T}=(0,1)$.

The above model admits classical vortex solutions: $Z$-strings and $W$-strings. Unlike vortices in the Abelian Higgs model, these "embedded" strings are not topological and they exist only for energetic rather than topological reasons. Their stability relies sensitively 
on the parameters of the theory. When the analysis is restricted to the bosonic sector, it has been shown that $Z$ strings are only stable for small Higgs mass and $\theta_{w}$ close to $\pi / 2$ [10,11] while $W$-string are always unstable [12]. A unit winding $Z$-string along the $z$ axis in cylindrical coordinates is a solution of the form

$$
\Phi=\frac{\eta}{\sqrt{2}} f(r) e^{i \theta}\left(\begin{array}{l}
0 \\
1
\end{array}\right), \quad Z_{\theta}=-\frac{2}{\alpha} \frac{v(r)}{r}
$$

with all other components and fields vanishing, where $f(r)$ and $v(r)$ satisfy:

$$
\begin{array}{r}
f^{\prime \prime}+\frac{f^{\prime}}{r}-\frac{f}{r^{2}}(1-v)^{2}+\lambda \eta^{2}\left(1-f^{2}\right) f=0 \\
v^{\prime \prime}-\frac{v^{\prime}}{r}+\frac{\alpha^{2} \eta^{2}}{4} f^{2}(1-v)=0 \\
f(0)=v(0)=0, \quad f(\infty)=v(\infty)=1
\end{array}
$$

Now we include the fermion sector of the theory. Without loss of generality, we consider only quarks in one generation.

$$
\begin{array}{r}
\mathcal{L}_{\text {Quark }}=\bar{\Psi}_{L} i \not D^{L} \Psi_{L}+\overline{u_{R}} i \not D^{R} u_{R}+\bar{d}_{R} i \not D^{R} d_{R} \\
-G_{u}\left(\bar{\Psi}_{L} \tilde{\Phi} u_{R}+\overline{u_{R}} \tilde{\Phi}^{\dagger} \Psi_{L}\right) \\
-G_{d}\left(\overline{\Psi_{L}} \Phi d_{R}+\bar{d}_{R} \Phi^{\dagger} \Psi_{L}\right)
\end{array}
$$

where

$$
\begin{aligned}
& \Psi_{L}=\left(\begin{array}{c}
u_{L} \\
d_{L}
\end{array}\right), \quad \tilde{\Phi}=i \tau_{2} \Phi^{*}=\left(\begin{array}{c}
\phi_{0}^{*} \\
-\phi_{1}^{*}
\end{array}\right) \\
& D_{\mu}^{L} \Psi_{L}=\left(\partial_{\mu}-\frac{i}{2} g \tau^{a} W_{\mu}^{a}-\frac{i}{2} g^{\prime} Y B_{\mu}\right) \Psi_{L} \\
& D_{\mu}^{R} \Psi_{R}=\left(\partial_{\mu}-\frac{i}{2} g^{\prime} Y_{R} B_{\mu}\right) \Psi_{R}
\end{aligned}
$$

In above $\Psi_{R}$ stands for $u_{R}$ or $d_{R}$ and $Y_{R}$ for their corresponding hypercharges.

The Dirac equations for the quark fields in the background of a straight $Z$-string $(2+1$ dimension) are:

$$
i \partial_{t}\left(\begin{array}{l}
u \\
d
\end{array}\right)=H_{0}\left(\begin{array}{l}
u \\
d
\end{array}\right)=\left(\begin{array}{cc}
H_{u u} & 0 \\
0 & H_{d d}
\end{array}\right)\left(\begin{array}{l}
u \\
d
\end{array}\right)
$$

with

$$
\begin{gathered}
u=\left(\begin{array}{l}
u_{L} \\
u_{R}
\end{array}\right), \quad d=\left(\begin{array}{l}
d_{L} \\
d_{R}
\end{array}\right), \quad H_{0}=\left(\begin{array}{cc}
H_{u u} & 0 \\
0 & H_{d d}
\end{array}\right) \\
H_{u u}=\gamma_{0}\left(\begin{array}{cc}
-i \phi_{u} & G_{u} \phi_{0}^{*} \\
G_{u} \phi_{0} & -i \phi_{u}
\end{array}\right), \quad H_{d d}=\gamma_{0}\left(\begin{array}{cc}
-i \phi_{d} & G_{d} \phi_{0} \\
G_{d} \phi_{0}^{*} & -i \phi_{d}
\end{array}\right)
\end{gathered}
$$




$$
\not_{u, d}=\gamma^{i}\left(\partial_{i}-i \alpha Z_{i} Q_{z}^{u, d}\right), \quad i=1,2
$$

where $Q_{z}$ are the $Z$ charge matrices for $u$ and $d$, namely,

$$
Q_{z}^{u, d}=\left(\begin{array}{cc}
q_{L}^{u, d} & 0 \\
0 & q_{R}^{u, d}
\end{array}\right)=\frac{1}{2}\left(\begin{array}{cc} 
\pm 1-(y \pm 1) \sin ^{2} \theta_{w} & 0 \\
0 & -(y \pm 1) \sin ^{2} \theta_{w}
\end{array}\right)
$$

with $y=Y\left(\Psi^{L}\right)$, the hypercharge of $u_{L}$ and $d_{L}$. Note that the above equations for $u$ and $d$ are not coupled.

Equation (3) has zero energy solutions ("zero modes") that are independent of $(z, t)$ and are normalizable in the $x y$ plane. Earlier analyses of zero modes on topological strings using index theorems [9] find precisely one zero mode per massive fermion on a string with unit winding number. This result holds in our case too and so there exists precisely one zero mode on a string with winding number 1 for each of $u$ and $d$. Furthermore, the states are eigenstates of the operator $\gamma^{0} \gamma^{z}$. As $u$ couples to $\phi_{0}$ (a vortex) while $d$ couples to $\phi_{0}^{*}$ (an antivortex), we have:

$$
\gamma^{0} \gamma^{z} u_{0}=u_{0}, \quad \gamma^{0} \gamma^{z} d_{0}=-d_{0}
$$

where, $u_{0}$ and $d_{0}$ denote the zero modes for the $u$ and $d$ fields. In the context of $3+1$ dimension, the zero energy solution above generates a whole family of solutions of Dirac equations of the form:

$$
\Psi_{+}=\beta_{+}(t, z) u_{0}(x, y), \quad \Psi_{-}=\beta_{-}(t, z) d_{0}(x, y)
$$

with

$$
\left(\partial_{t} \pm \partial_{z}\right) \beta_{ \pm}(t, z)=0
$$

We may think of these solutions as massless chiral fermions trapped on the string moving at the speed of light: up quarks move along the string in one direction and we will refer to them as "right-movers" and down quarks move along the string in the opposite direction and will be called "left-movers".

\section{OUTLINE AND SCOPE OF CALCULATION}

The mutual interaction of fermions and solitons has been studied over the last few decades and a number of excellent reviews exist (for example, see Chapter 9 of [13). In this section, we would like to summarize a standard technique for studying the interaction of fermions with solitons. Our motivation for this summary is so that we can indicate the difficulties likely to be encountered in doing the full calculation and hence indicate the scope of the present calculation.

The basic idea is that, for a renormalizable theory, Fermi fields always enter bilinearly in the Lagrangian and can be integrated out of the functional integral yielding an effective action for the bosonic fields. If the fermionic part of the action is written in terms of an operator $K\left[\Phi, A_{\mu}\right]$ as:

$$
\int d^{4} x i \Psi^{\dagger} K\left[\Phi, A_{\mu}\right] \Psi
$$


then the effective action for time-independent bosonic fields is:

$$
S_{e f f}\left[\Phi, A_{\mu}\right]=S_{B}\left[\Phi, A_{\mu}\right]-i \ln \operatorname{Det} K\left[\Phi, A_{\mu}\right] .
$$

Thus the contributions of the Fermi fields have been entirely absorbed in the second term and the semiclassical bosonic solution with fermion back reaction can be obtained by extremizing $S_{\text {eff }}$ :

$$
\delta S_{\text {eff }}\left[\Phi, A_{\mu}\right]=\delta\left\{S_{B}-i \ln \operatorname{Det} K\left[\Phi, A_{\mu}\right]\right\}=0
$$

The effects of the Fermi fields become more transparent when $\operatorname{Det} K\left[\Phi, A_{\mu}\right]$ is expressed formally as:

$$
\operatorname{Det} K\left[\Phi, A_{\mu}\right]=\sum_{\left\{n_{r}\right\}} C\left(\left\{n_{r}\right\}\right) \exp \left[-i T\left(\sum_{r}\left(-\epsilon_{r}+n_{r} \epsilon_{r}\right)\right)\right]
$$

where $\epsilon_{r}=\epsilon_{r}\left[\Phi, A_{\mu}\right]$ are the eigenvalues of the one particle Dirac Hamiltonion in the presence of $\Phi$ and $A_{\mu}, n_{r}$ are the occupation numbers for exited states and $C\left(\left\{n_{r}\right\}\right)$ are the combinatoric degeneracy factors. We recognize that the ground state (with all $n_{r}=0$ ) energy $E_{0}=-\sum_{r} \epsilon_{r}$ is just the familiar filled "Dirac sea".

In practice, it is almost always impossible to implement the above scheme because $\ln \operatorname{Det} K\left[\Phi, A_{\mu}\right]$ in $S_{\text {eff }}$ is, in general, a non-polynomial non-local functional and its explicit form is unobtainable. Instead, to order $\hbar$, it is sufficient to evaluate the ground state $(g s)$ energy of the classical bosonic configuration plus fermionic vacuum as:

$$
E_{g s}=E_{0}+\left(E_{b}+\sum_{r}\left(-\epsilon_{r}\right)-E_{c t}\right)
$$

where, $E_{0}$ is the energy of the classical bosonic configuration, $E_{b}$ is the contribution due to bosonic fluctuations, the sum is over all filled energy levels (Dirac sea) with $\epsilon_{r}$ being the energy eigenvalue of the $r^{\text {th }}$ level in the background of the classical bosonic configuration and $E_{c t}$ is the energy contribution coming from the counterterms that are necessary to cancel off the divergences.

In our case, therefore, we would like to calculate all the fermionic energy levels in the background of the $Z$-string and the energy contribution from the counterterms. That will yield $E_{g s}-E_{b}$. Then we would like to do the same calculation with a slightly perturbed $Z$-string; this will yield $E_{g s}-E_{b}+\delta E$. If $\delta E$ is negative, the fermionic vacuum destabilizes the string; if it is positive, we say that the fermionic vacuum tends to stabilize the string.

This simplified calculation is also beyond our reach for we do not know all the energy levels $\epsilon_{r}$. We only know a part of the spectrum - the zero modes on the string. If we assume that the dominant contribution to the term in parenthesis in (9) comes from the zero modes we might be able to say something about the effects of fermions on the string configuration!

\footnotetext{
${ }^{1}$ Intuitively it seems reasonable that a fermion scattering state should give a smaller contribution to $\delta E$ than a bound state. This is because the scattering states live outside the string core and have little overlap with perturbations of the string core. But it is not known if the zero modes are the only bound states and if the summed effect of all the scattering states is smaller than the summed effect of the bound states since there are many more scattering states than there are bound states.
} 
Indeed this calculation was performed in $\llbracket$ with the conclusion that $\delta E$ due to the fermionic vacuum is always negative. We will outline this calculation in Sec. VI and argue that this probably leads to a distortion of the $Z$-string.

\section{PERTURBED ZERO MODES IN 2+1 DIMENSION}

In the case of topological strings, the existence of fermionic zero modes follows from an index theorem. Then it is clear that the zero modes will continue to exist even if the topological string solution is perturbed. For the same reason, since the $Z$-string is a topological string when we restrict ourselves to the $U(1)$ sector defined by the $Z$ gauge field, the zero modes continue to exist when only the $\phi_{0}, Z$ and $A$ fields are perturbed. (A simple explicit argument to see this without recourse to the index theorem is described in the Appendix.) However, in the full model, perturbations involving $W^{ \pm}$and $\phi^{+}$fields take one out of the $U(1)$ sector and we may expect non-trivial consequences for the zero modes.

According to [10], the physical modes that destabilize the $Z$-string involve combinations of $W_{ \pm}$and $\phi_{1}$ modes in the $x y$ plane. Therefore, we will restrict our discussions to perturbation of this type, i.e. we assume

$$
\phi_{1}=\epsilon \phi_{1}(x, y), \quad W^{\mu}=\epsilon\left(0, W^{1}(x, y), W^{2}(x, y), 0\right)
$$

where $\phi, W^{1}, W^{2}$ are arbitrary complex functions of $x$ and $y$ and $\epsilon$ is a perturbation parameter. Note that we have not fixed the gauge as this is not necessary for our purposes. (For an alternate approach to the stability analysis see [11].)

In $(2+1)$ dimension, with $\Psi=\psi(x, y) e^{-i E t}$, the Dirac equations for quarks in the background of the perturbed string become energy eigenvalue equations:

$$
H \Psi=E \Psi
$$

with

$$
\begin{gathered}
\Psi=\left(\begin{array}{l}
u \\
d
\end{array}\right), \quad H=H_{0}+\epsilon H_{1}, \quad H_{1}=\left(\begin{array}{cc}
0 & H_{u d} \\
H_{d u} & 0
\end{array}\right) \\
H_{u d}=H_{d u}^{\dagger}=\gamma_{0}\left(\begin{array}{cc}
-\frac{g}{2} W^{+} & G_{d} \phi_{1} \\
-G_{u} \phi_{1} & 0
\end{array}\right) \quad W^{ \pm}=W^{1} \mp i W^{2} \quad W^{+}=\gamma^{\mu} W_{\mu}^{+}
\end{gathered}
$$

where $u, d, H_{0}$ have the same meaning as in (国), (5), (6) and $\phi_{1}, W^{ \pm}$are given by (10). Notice that the perturbations in $W$ and $\phi_{1}$ introduce couplings between the $u$ and $d$ fields.

A crucial property of the Hamiltonian, and one which we will repeatedly use, is:

$$
\left\{\gamma^{0} \gamma^{z}, H\right\}=0
$$

where \{\} stands for the anticommutator. The origin of this property can be traced to the two dimensional character of the field configuration and its existence can be expected in any

\footnotetext{
${ }^{2}$ The leptons can be similarly considered.
} 
generic $(2+1)$ dimensional problem. In our case, (13) is true both before and after the string is perturbed. From (13), the transformation: $\Psi \rightarrow \gamma^{0} \gamma^{z} \Psi$ (called "particle conjugation" in [14]) takes a solution with energy $E$ to one with energy $-E$, i.e. if

$$
H \Psi=E \Psi
$$

then

$$
H\left(\gamma^{0} \gamma^{z} \Psi\right)=-E\left(\gamma^{0} \gamma^{z} \Psi\right)
$$

Also, since $H$ is hermitian, $\Psi$ and $\gamma^{0} \gamma^{z} \Psi$ are orthogonal to each other except when $E=0$.

Now we investigate the behaviour of the zero modes in the context of perturbation theory. When $\epsilon=0$, we recover (3), where the equations for $u$ and $d$ are uncoupled and there are two degenerate zero modes

$$
\left(\begin{array}{c}
u_{0} \\
0
\end{array}\right), \quad\left(\begin{array}{c}
0 \\
d_{0}
\end{array}\right) .
$$

When the string is perturbed, the energy eigenstates are linear combinations of $\left(u_{0}, 0\right)^{T}$ and $\left(0, d_{0}\right)^{T}$ and so we write them as:

$$
\Psi_{0}=a\left(\begin{array}{c}
u_{0} \\
0
\end{array}\right)+b\left(\begin{array}{c}
0 \\
d_{0}
\end{array}\right)
$$

where, $a$ and $b$ are constants that need to be determined. The equation for $\Psi_{0}$ is

$$
H \Psi_{0}=\left(H_{0}+\epsilon H_{1}\right) \Psi_{0}=\left(E_{0}+\epsilon E_{1}\right) \Psi_{0}
$$

and is straightforward to solve. This gives:

$$
E_{1}= \pm\left|m_{1}\right|, \quad \frac{a}{b}= \pm \frac{m_{1}}{\left|m_{1}\right|} \equiv \pm e^{i \beta}
$$

where $m_{1}$ is defined by

$$
m_{1} \equiv<u_{0}\left|H_{u d}\right| d_{0}>=\int d^{2} x u_{0}^{\dagger} H_{u d} d_{0}
$$

The magnitude of $a$ and $b$ is not fixed by perturbation theory but can be fixed by imposing a suitable normalization condition.

Eq. (17) shows that the perturbations on the string lift the two-fold degeneracy of zero modes yielding two states with opposite energies. In the limit that the perturbation is turned off $(\epsilon \rightarrow 0)$, the two eigenstates with their $H_{1}$ eigenvalues are:

$$
\begin{gathered}
\Psi_{0}=\left(\begin{array}{c}
u_{0} \\
e^{-i \beta} d_{0}
\end{array}\right), \quad E_{1}=+\left|m_{1}\right| \\
\tilde{\Psi}_{0}=\left(\begin{array}{c}
u_{0} \\
-e^{-i \beta} d_{0}
\end{array}\right), \quad \tilde{E}_{1}=-\left|m_{1}\right|
\end{gathered}
$$


Note that $\tilde{\Psi}_{0}$ and $\Psi_{0}$ satisfy

$$
\tilde{\Psi}_{0}=\gamma^{0} \gamma^{z} \Psi_{0}, \quad \tilde{E}_{1}=-E_{1}
$$

a consequence of (14) and (15). Actually, (14) and (15) require that the relation (21) holds to all orders in perturbation theory, i.e. if we denote the set of corrections to the wave function and energy of $\Psi_{0}$ as $\left\{\Psi_{n}, E_{n}, n=1,2, \ldots\right\}$ and that of $\tilde{\Psi}_{0}$ as $\left\{\tilde{\Psi}_{n}, \tilde{E}_{n}, n=1,2, \ldots\right\}$, then

$$
\tilde{\Psi}_{n}=\gamma^{0} \gamma^{n} \Psi_{n}, \quad \tilde{E}_{n}=-E_{n}
$$

for all $n$.

In analyzing the effect of the fermions on the stability of the string, it is necessary to work up to second order in perturbation theory. So we now need to find $E_{2}$.

First note that the conventional result for the second order change in energy of a state $n>$ is written as:

$$
E_{2}^{(n)}=\sum_{m \neq n} \frac{|<m| H^{\prime}|n>|^{2}}{E_{0}^{(m)}-E_{0}^{(n)}}
$$

where, $E_{0}^{(i)}$ denotes the energy of the unperturbed $i^{\text {th }}$ level. This sum requires knowledge of the entire unperturbed spectrum $E_{0}^{(i)}$ and hence cannot be used in our case where we only know one state, namely, the zero mode. So we need to find $E_{2}$ by some other means. The means we adopt is to make crucial use of the property in (13).

Let us denote the perturbative corrections to the wave function and energy of the zero mode by $\left\{\Psi_{n}, E_{n}, n=1,2, \ldots\right\}$ and that of $\tilde{\Psi}_{0}$ as $\left\{\tilde{\Psi}_{n}, \tilde{E}_{n}, n=1,2, \ldots\right\} . E_{2}$ (similarly $\tilde{E}_{2}$ ) can be obtained by:

$$
E_{2}=<\Psi_{0}\left|H_{1}\right| \Psi_{1}>
$$

but we first have to find $\Psi_{1}$. If we write

$$
\Psi_{1}=\left(\begin{array}{c}
u_{1} \\
d_{1}
\end{array}\right)
$$

then $u_{1}$ and $d_{1}$ satisfy:

$$
\begin{aligned}
H_{u u} u_{1} & =b m_{1} u_{0}-b H_{u d} d_{0} \\
H_{d d} d_{1} & =a m_{1}^{*} d_{0}-a H_{d u} u_{0}
\end{aligned}
$$

and,

$$
E_{2}=a^{*}<u_{0}\left|H_{u d}\right| d_{1}>+b^{*}<d_{0}\left|H_{d u}\right| u_{1}>
$$

Equations (24) and (25) are impossible to solve (even numerically) since they involve the unspecified perturbations $W^{\mu}$ and $\phi^{+}$entering via $m_{1}, H_{u d}$ and $H_{d u}$. Yet, as we show below, it is still possible to find $E_{2}$ (and $\tilde{E}_{2}$ ) by using the property in (13).

Let 


$$
u_{1}=u_{1}^{+}+u_{1}^{-}, \quad d_{1}=d_{1}^{+}+d_{1}^{-}
$$

where

$$
\gamma^{0} \gamma^{z} u_{1}^{ \pm}= \pm u_{1}^{ \pm}, \quad \gamma^{0} \gamma^{z} d_{1}^{ \pm}= \pm d_{1}^{ \pm}
$$

Then, using the property in (13) together with $\left(\gamma^{0}\right)^{\dagger}=\gamma^{0}$ and $\left(\gamma^{z}\right)^{\dagger}=-\gamma^{z}$, we get,

$$
<u_{0}\left|H_{u d}\right| d_{1}^{+}>=-<u_{0}\left|\left(\gamma^{0} \gamma^{z}\right)^{\dagger} H_{u d}\left(\gamma^{0} \gamma^{z}\right)\right| d_{1}^{+}>
$$

Now we use eqns. (7) and (28) which give:

$$
<u_{0}\left|H_{u d}\right| d_{1}^{+}>=0
$$

Similarly, we find

$$
<d_{0}\left|H_{d u}\right| u_{1}^{-}>=0
$$

Then (26), (30) and (31) give

$$
E_{2}=a^{*}<u_{0}\left|H_{u d}\right| d_{1}^{-}>+b^{*}<d_{0}\left|H_{d u}\right| u_{1}^{+}>
$$

Note that $u_{1}^{-}$and $d_{1}^{+}$do not contribute to $E_{2}$ (or to $\tilde{E}_{2}$ ). In terms of $u_{1}^{ \pm}, d_{1}^{ \pm}$, equations (24) and (25) reduce to:

$$
\begin{aligned}
H_{u u} u_{1}^{+}=0, & H_{u u} u_{1}^{-}=b m_{1} u_{0}-b H_{u d} d_{0} \\
H_{d d} d_{1}^{-}=0, & H_{d d} d_{1}^{+}=a m_{1}^{*} d_{0}-a H_{d u} u_{0}
\end{aligned}
$$

We see that the equations for $u_{1}^{+}$and $d_{1}^{-}$are just the zero modes equations of $H_{0}$ (see (3)) and so the solutions are $u_{1}^{+} \propto u_{0}$ and $d_{1}^{-} \propto d_{0}$. But since we have fixed the normalization of the wave-function, we require $<\Psi_{0} \mid \Psi_{1}>=0$. Therefore $u_{1}^{+}$and $d_{1}^{-}$have to vanish. From (32) (and similarly for $\tilde{E}_{2}$ ) we then get

$$
E_{2}=\tilde{E}_{2}=0
$$

\section{PERTURBED ZERO MODES IN 3+1 DIMENSION}

In a $(3+1)$ dimensional context, equation (11) becomes:

$$
\left(H-i \gamma^{0} \gamma^{z} \partial_{z}\right) \Psi=E \Psi
$$

where $H=H(x, y)$ is defined as before with no dependence on $z$. In this case, it is straightforward to obtain the perturbed zero modes moving along the string by Lorentz boosting the perturbed solutions in $(2+1)$ dimension that we found in the last section along the $z$ direction. Considering only the lowest order correction, we get two sets of levels:

$$
E=\sqrt{P^{2}+\epsilon^{2}\left|m_{1}\right|^{2}}
$$


by boosting $\Psi_{0}$ and

$$
\tilde{E}=-\sqrt{P^{2}+\epsilon^{2}\left|m_{1}\right|^{2}}
$$

by boosting $\tilde{\Psi}_{0}$, where, $P$ is the momentum in the $z$ direction. This is just the energy spectrum for a particle moving freely along the string with momentum $P$ and mass $\epsilon\left|m_{1}\right|$.

Note that we only need to boost the first order correction to the zero mode $(P=0)$ in order to get the second order correction to the $P \neq 0$ modes. Strictly, this follows only for $P^{2}>>\epsilon^{2}\left|m_{1}\right|^{2}$. This assumption is justified if we assume periodic boundary conditions in the $z$ direction with period $L$ and assume $\epsilon$ small enough so that $\epsilon m_{1} L<<1$. (For an infinite string, we need to take the limit $L \rightarrow \infty$ as well as $\epsilon \rightarrow 0$ for perturbation theory to be applicable. The necessity of taking two limits requires care and we always assume that they are taken so that $\epsilon\left|m_{1}\right| L$ remains small.)

The above results for $E$ and $\tilde{E}$ can also be reached by considering perturbations around the massless modes in (8). Since

$$
\left[H, P_{z}\right]=0
$$

we can choose $\Psi$ to be an eigenstate of $P_{z}$, i.e.

$$
\Psi \rightarrow e^{i P z} \Psi(t, x, y)
$$

Our unperturbed massless states are:

$$
\begin{array}{ll}
E_{0}=P, & \Psi_{0}=e^{i\left(-E_{0} t+P z\right)}\left(\begin{array}{c}
u_{0} \\
0
\end{array}\right) \\
E_{0}=-P, & \Psi_{0}=e^{i\left(-E_{0} t+P z\right)}\left(\begin{array}{c}
0 \\
d_{0}
\end{array}\right)
\end{array}
$$

Now let us look at the perturbations of the above states when the strings are perturbed. (We will only discuss the case $E_{0}=P$; identical procedures apply to the case $E_{0}=-P$.)

Notice that because $P_{z}$ and $H$ commute (eq. (35)), we can restrict our attention to sectors of fixed $P_{z}$ when doing the perturbation analysis. In such a sector with $P \neq 0$, the zeroth order wave functions are not degenerate. Now since $H_{1}$ (see (12)) is off diagonal, and the zeroth order wave function with $E_{0}=+P, \Psi_{0}$, is proportional to $\left(u_{0}, 0\right)^{T}$, the first order energy perturbation $E_{1}=<\Psi_{0}\left|H_{1}\right| \Psi_{0}>=0$.

The energy perturbation will be non-trivial in the second order calculation but, as in the $(2+1)$ dimensional case, the standard result of second order perturbation theory cannot be used here. Once again this difficulty can be avoided by the same technique used in Sec. IV. Using the same notation as in the $(2+1)$ dimensional case (see (23)), now the equations for $u_{1}$ and $d_{1}$ become:

$$
\begin{aligned}
H_{u u} u_{1} & =P\left(1-\gamma^{0} \gamma^{z}\right) u_{1} \\
H_{d d} d_{1} & =P\left(1-\gamma^{0} \gamma^{z}\right) d_{1}-H_{d u} u_{0}
\end{aligned}
$$

Decomposing $u_{1}$ and $d_{1}$ as in (27) and (28), the above equations become: 


$$
\begin{array}{ll}
H_{u u} u_{1}^{-}=0, & H_{u u} u_{1}^{+}=2 P u_{1}^{-} \\
H_{d d} d_{1}^{-}=0, & H_{d d} d_{1}^{+}=2 P d_{1}^{-}-H_{d u} u_{0}
\end{array}
$$

Since $H_{u u}$ does not have any zero energy solution which is also an eigenstate of $\gamma^{0} \gamma^{z}$ with eigenvalue -1 , it follows from (36) that $u_{1}^{-}=0$, and the equation for $u_{1}^{+}$again reduces to that of the zero modes. With the imposition of the normalization condition, we conclude $u_{1}^{+}=0$.

Next let us look at (37). The equation for $d_{1}^{-}$leads to:

$$
d_{1}^{-}=N d_{0}
$$

where $N$ is some constant to be determined. (Note that the normalization condition < $\Psi_{0} \mid \Psi_{1}>=0$ does not impose any constraint on $N$.) Plugging the above expression for $d_{1}^{-}$into the equation for $d_{1}^{+}$, taking the inner product of the equation with $d_{0}$ and using $H_{d d} d_{0}=0$ (see (3)), we get:

$$
2 P N=<d_{0}\left|H_{d u}\right| u_{0}>\Rightarrow N=\frac{m_{1}^{*}}{2 P} \Rightarrow d_{1}^{-}=\frac{m_{1}^{*}}{2 P} d_{0}
$$

where $m_{1}$ is defined as before (see (18)). Then, it follows that:

$$
E_{2}=<\Psi_{0}\left|H_{1}\right| \Psi_{1}>=<u_{0}\left|H_{1}\right| d_{1}^{-}>=\frac{\left|m_{1}\right|^{2}}{2 P} .
$$

Therefore our final results are:

$$
E=E_{0}+\epsilon E_{1}+\epsilon^{2} E_{2}=P+\epsilon^{2} \frac{\left|m_{1}\right|^{2}}{2 P}
$$

for left-movers, and,

$$
E=E_{0}+\epsilon E_{1}+\epsilon^{2} E_{2}=-P-\epsilon^{2} \frac{\left|m_{1}\right|^{2}}{2 P}
$$

for right-movers. These expressions agree with (33) and (34) to second order in $\epsilon^{2}$ for $P^{2}>>\epsilon^{2}\left|m_{1}\right|^{2}$. We exhibit these results in Fig. 1 .

In [4], the results in (39) and (40) were also obtained but by applying the standard formula of second order perturbation theory (22).

\section{CONCLUSIONS AND DISCUSSION}

We have explored the effects of perturbations on $Z$-strings on fermion zero modes in $2+1$ and $3+1$ dimensions. For the $P=0$ state, we used degenerate perturbation theory to find the first order correction to the energy of the zero mode. A difficulty which we encountered when trying to find the second order corrections to the energy (for any value of $P)$ was the unavailability of a complete basis of zeroth order wave functions. The standard formula of second order perturbation theory assumes the knowledge of such a basis. We circumvented this difficulty by using symmetry arguments and by extracting the relevant 
part of the solution to the first order equations. This then gave us the second order correction to the energy for all values of $P$.

Having found the effect that perturbations on electroweak strings have on fermion zero modes we now wish to discuss the backreaction of fermions on the string. This problem has been discussed by Naculich [1] and we first summarize his result.

The fermionic ground state consists of the Dirac sea in which all negative energy levels are filled. Then the effect of the perturbations can be found by summing the shifts in all the negative energy levels. From (40), this leads to a sum of the kind:

$$
\Delta E=-\frac{\epsilon^{2}}{2}\left|m_{1}\right|^{2} L \sum_{n=1}^{N} \frac{1}{n}
$$

where periodic boundary conditions have been imposed along the string with period $L$, an ultraviolet cut-off on the sum has been imposed and the two degenerate states with $P=0$ have been taken to be both filled or both empty. The sum in (41) is logarithmically divergent. Furthermore, the shift in the energy is negative since all the negative energy modes have shifted down (see Fig. 1). The divergence in this contribution will be tamed once the contribution of the counterterms (see eq. (91)) is taken into account but the sign remains negative for an infinite string. This indicates that the fermionic vacuum destabilizes the bosonic electroweak string. As discussed in [4], this could mean that the solution for the string - found by solving the classical equations in the bosonic sector - gets distorted by the fermionic vacuum or it might imply that there is a runaway instability and the string decays into the vacuum. We now argue that this instability is likely to distort the string but not lead to decay.

The instability discussed above relies on three features of the system on hand. These are: (i) the existence of fermionic zero modes on the unperturbed strings, (ii) the existence of certain perturbations that give the massless modes a small mass as in eqs. (33) and (34), and, (iii) the logarithmic divergence in the sum over the Dirac sea. As these features are present in a wide variety of defects, the fermionic vacuum instability can be expected to apply in very general situations. In particular, suppose we have a model in which topological magnetic monopoles are produced at extremely high energy scales such as near the Planck scale and then get connected by strings at a low energy scale such as the electroweak scale. Then, in the low energy theory, the strings are the usual topological strings and we can consider a fermionic sector such that fermionic zero modes exist on these strings. This model has all the features necessary for the fermionic vacuum instability. By assumption, it has the feature that there are fermionic zero modes on the strings. This implies that the fermions are massive outside the string and massless within. But then, since the strings can terminate, the distortions of the string that are present near the terminus (monopole) must give a mass to the fermions exactly as in (33) and (34). Once this feature is present, the logaritmic divergence with the negative sign simply follows from summing over the Dirac sea exactly as in (41). This shows that even when the monopoles are extremely heavy (Planck scale) and the strings are very light (electroweak scale), the fermionic vacuum instability persists. However, in this case, we know that the only way the string can decay is by nucleating monopole-antimonopole pairs because of the topology in the model. Given that the monopole is very heavy, the nucleation is an exponentially suppressed quantum tunneling process. So the instability due to the fermionic vacuum cannot lead to string decay but can 
only distort the string configuration.

The electroweak string can also be viewed as a topological string that can terminate on monopoles [5] except that there is no vast separation of scales as in the above example. But this separation of scales does not enter the fermionic vacuum instability and is irrelevant for present purposes. So we expect that the fermionic vacuum contributions found in [4] should lead to a distortion of the electroweak string but not be responsible for decay.

Another argument that supports this conjecture comes from the fact that the instability does not depend on whether the string is global or local and so it should also apply in condensed matter systems where fermionic zero modes exist on global strings. ${ }^{3} \mathrm{He}$ is one such system in which topologically unstable global strings with fermionic zero modes exist [6] and such strings have been observed in the laboratory. It has been argued [7] that the fermionic vacuum should distort the structure of these strings. Perhaps we will be able to observe the fermionic vacuum distortion of strings in ${ }^{3} \mathrm{He}$ in the near future.

\section{Acknowledgements}

We are grateful to Steve Naculich and Grisha Volovik for enlightening discussions. 


\section{REFERENCES}

[1] Y. Nambu, Nucl. Phys. B 130 (1977) 505.

[2] T. Vachaspati, Phys. Rev. Lett. 68 (1992) 1977-1980, ibid 69 (1992) 216E.

[3] M. Earnshaw and W. Perkins, Phys. Lett. B 328 (1994) 337.

[4] S. Naculich, Phys. Rev. Lett. 75 (1995) 998-1001; S. Kono and S. Naculich, hepph/9507350.

[5] T. Kephart and T. Vachaspati, hep-ph/9503355.

[6] G.E. Volovik and T. Vachaspati, cond-mat/9510065.

[7] Y.G. Makhlin and G.E. Volovik, cond-mat/9510075.

[8] G.E. Volovik, private communication.

[9] E. Weinberg, Phys. Rev. D 24 (1981) 2669.

[10] M. James, L. Perivolaropoulos and T. Vachaspati, Nucl. Phys. B 395 (1993) 534-546.

[11] M. Goodband and M. Hindmarsh, hep-ph/9505357.

[12] M. Barriola, T. Vachaspati and M. Bucher, Phys. Rev. D 50, (1994) 2819-2825.

[13] R. Rajaraman, Solitons and Instantons North-Holland (1982).

[14] R. Jackiw and P. Rossi, Nucl. Phys. B 190 (1980) 681. 


\section{Appendix}

We now give a simple explicit proof that the zero modes on Nielsen-Olesen vortices in the Abelian Higgs model continue to exist even when the vortices undergo deformations (which are not necessarily small). The proof is based on the property (13) of the Hamiltonian.

Consider an eigenstate $\psi$ of the Dirac Hamiltonian in a deformed string background. Then we write

$$
H=H_{0}+\epsilon H_{1}
$$

where $H_{0}$ is the Hamiltonian in the undeformed string background, $\epsilon$ is a parameter (not necessarily small) and $\epsilon H_{1}$ is the Hamiltonian due to the deformations of the string. Then we have

$$
H \psi=E \psi
$$

Next define:

$$
\psi_{0} \equiv \lim _{\epsilon \rightarrow 0} \psi
$$

We will first show that $\psi_{0}$ cannot be an eigenstate of the operator $\gamma^{0} \gamma^{z}$ if $E \neq 0$.

Eqns. (14) and (15) and continuity in the parameter $\epsilon$ give

$$
<\psi_{0}\left|\gamma^{0} \gamma^{z} \psi_{0}>=\lim _{\epsilon \rightarrow 0}<\psi\right| \gamma^{0} \gamma^{z} \psi>=0 .
$$

This says that the states $\psi_{0}$ and $\gamma^{0} \gamma^{z} \psi_{0}$ are orthogonal. But if $\psi_{0}$ were an eigenstate of $\gamma^{0} \gamma^{z}$ with eigenvalue $c$ we would have,

$$
<\psi_{0}\left|\gamma^{0} \gamma^{z} \psi_{0}>=c<\psi_{0}\right| \psi_{0}>=c
$$

which contradicts the orthogonality?.

In the Abelian Higgs model, as long as left- and right-moving fermions do not couple directly in the fermionic sector of the Lagrangian, $\psi_{0}$ - being either a left-mover or a rightmover - is necessarily an eigenstate of $\gamma^{0} \gamma^{z}$ and, hence, $E=0$ for all values of $\epsilon$. In the electroweak model, the left- and right-movers (down and up type quarks) do not couple directly if we restrict ourselves to the $Z$ sector of the model. And so, the zero modes will persist when the string is deformed within the $Z$ sector. However, once we allow perturbations outside the $Z$ sector, the left- and right-movers do couple directly via terms such as $\bar{u}_{L} \phi_{1} d_{R}$ and we can expect the zero modes to develop a mass. This is also clear from eqns. (19) and (20), where we see that $\psi_{0}$ is not an eigenstate of $\gamma^{0} \gamma^{z}$.

\footnotetext{
${ }^{3}$ Note that $c$ cannot vanish because $\gamma^{0} \gamma^{z}$ is unitary.
} 


\section{Figure Caption}

1. The spectrum of massless quarks on electroweak strings (straight lines) and when the strings are perturbed (hyperbolae). 


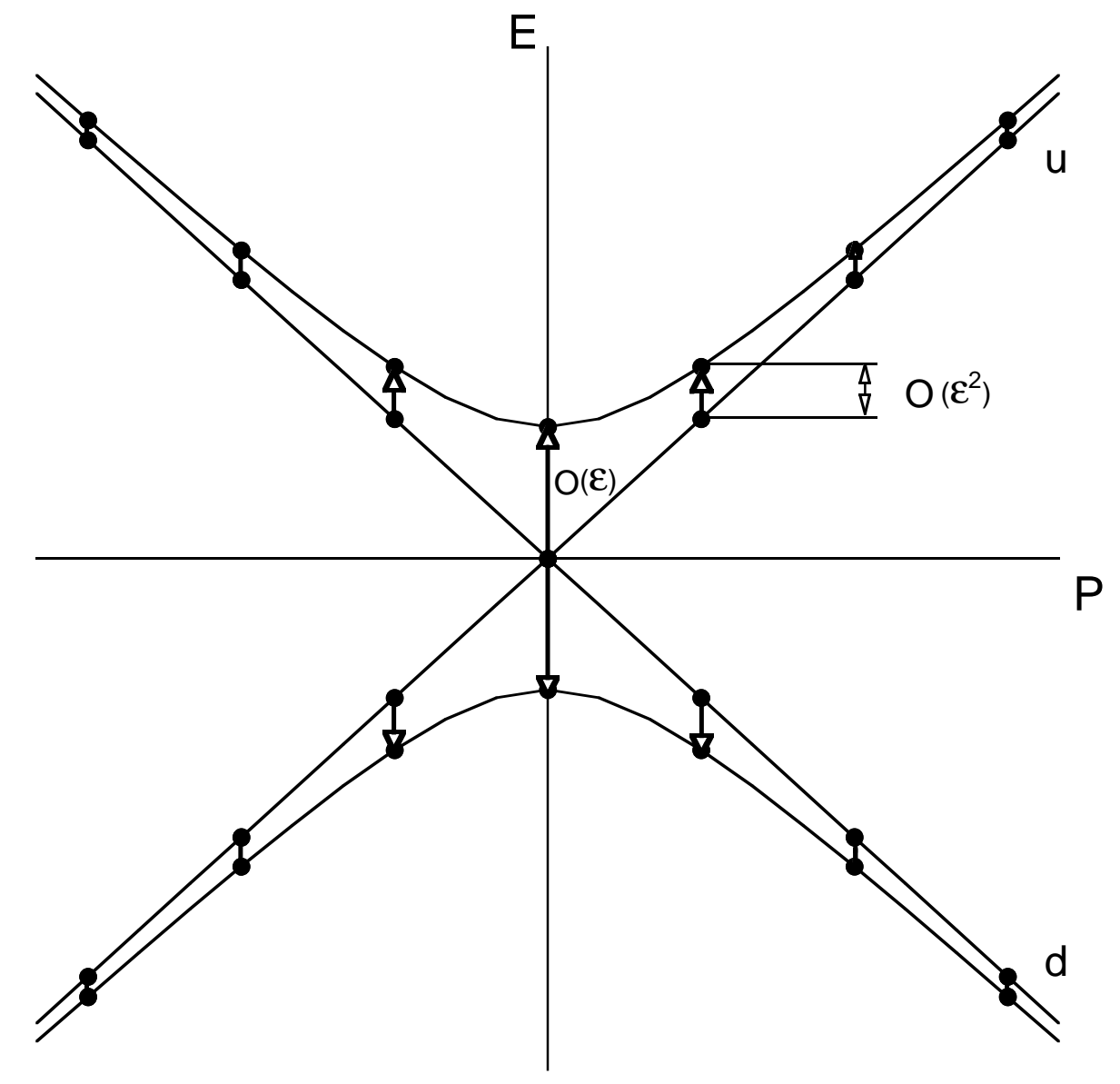

